\title{
Using Natural Language Processing to Translate Plain Text into Pythonic Syntax in Kannada
}

\section{Vinay Rao ${ }^{1 *}$, Sanjana GB ${ }^{2}$, Sundar Guntnur ${ }^{2}$, Navya Priya $\mathbf{N}^{2}$, Sanjana Reddy $^{2}$ and Pavan KR ${ }^{2}$}

${ }^{1}$ Independent Researcher, India

${ }^{2}$ RVCE, Mysore Rd, RV Vidyaniketan, Post, Bengaluru, Karnataka, India
Received: March 10, 2021

Published: April 26, 2021

(C) All rights are reserved by Vinay Rao., et al.

*Corresponding Author: Vinay Rao, Independent Researcher, Bangalore, India.

\begin{abstract}
Digital evolution has made various services and products available at everyone's fingertips and made human lives easier. It has become necessary for individuals with a passion to be a part of this digital evolution to learn how to write code, which is the basic literacy of the digital age. But writing code has become a privilege for students with prior knowledge of English.

In the context of the evolving field of Agri-tech, individuals and companies are making large strides towards digitising various different aspects of Agriculture. AI is being used actively to solve various problems in the agricultural space. The basic expected literacy here as well, is the ability to write code with the default understanding of English. Rural areas where one of the mainstream occupation for a large part of the population is Agriculture, English language may not be their primary language of choice for written and verbal communications.

With our work, we wish to provide a learning interface that users can employ to first learn the basics of writing code in their native language (Kannada will be in focus in our paper) and in future, the farmers can themselves build and consume tools that help them in their day to day needs with the skill of writing code that they can now acquire without the pre-requisites of knowing English.

The current model can successfully identify and convert conditional statements in the Kannada language into python code. The next effort will be aimed at extending this to recognise loop statements and create a framework for a wide variety of languages.
\end{abstract}

Keywords: Parts of Speech Tagger (PoS); Programming Languages (PL); Transfer Learning; AWD LSTM; fast.ai; Stemmer; Python; Kannada (Native South Indian Language)

\section{Introduction}

Digital technology has made revolutionary changes in human life. It plays an important role in almost every aspect of society. It has helped in the development of education, communication, agriculture, disaster response and many other fields. It helps in the economic growth of a country by improving efficiency.

Coding is the language in this modern digital world. It has become a centre of all business. Learning to code has become essential for anyone to pursue a career in this field. Many languages are available in which coding can be done. A survey shows that of 8500 programming languages available, 2400 were made in the United
States, 600 in the United Kingdom, 160 in Canada and 75 in Australia, English is native to these countries. Accordingly, more than one-third of these originated in English-speaking countries. Most of the resources available to learn and understand these languages are also in English [8].

But according to World Language Statistics (SIL International, 2015), English is the 3rd most spoken language in the world, with $5.43 \%$ of speakers, behind Chinese and Spanish with $14.4 \%$ and $6.15 \%$, respectively. And another survey of the most used Programming Languages' (TIOBE Software BV, 2019) Syntax, Semantics, Standard Library and Runtime System indicates that the most popular are all English based [11]. 
Even though Non-English-based PLs exist (Miller, Vandome, and McBrewster, 2012), currently the most used have syntax, learning resources, Runtime, and Development Environments that are developed with an English speaking audience in mind [11].

During the month of April of 2015, a survey was conducted on 78 students of the University College of Engineering, Osmania University. The survey was to perceive the importance of native language to understand the source code and the importance of comments in programs to understand the code. Figure 1 and 2 represent the output of the survey [11].

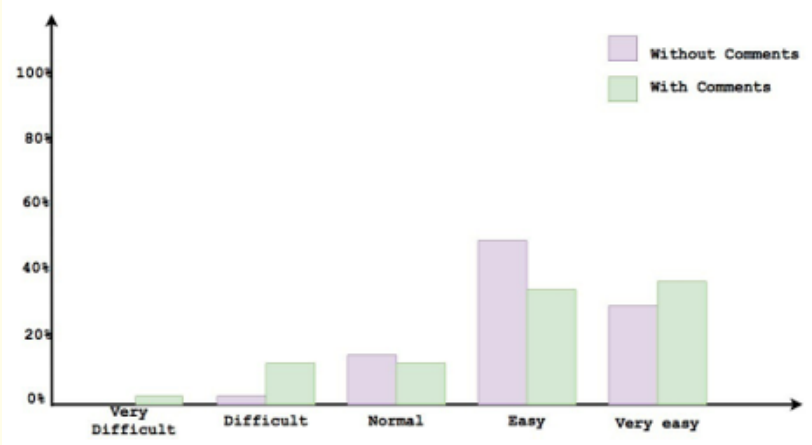

Figure 1: Result of the Survey to check the importance of comments in understanding the source code [11].

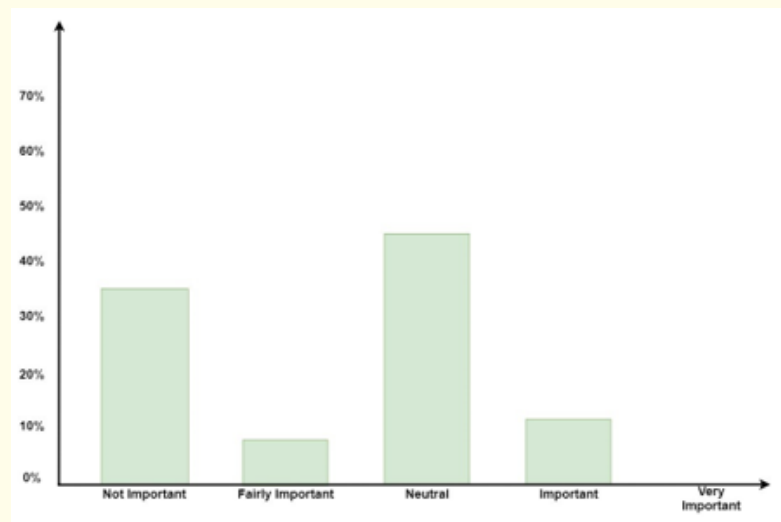

Figure 2: Result of the Survey to check the importance of native language in understanding the source code [11].

From the above survey, it was found that not only do many students find native language important or are neutral towards it, but the source code in the form of comment helps students learn the programming language. This survey highlights the need of the hour which is to make code more accessible to users who may not have
English as their primary mode of communication. This survey also throws light on the fact that code when expressed in the form of comments (plain text) does help in narrowing down the logic of what was implemented without needing the user to dig into the syntactic nuances of the coding language.

Therefore, in this paper, we present a learning platform where simple coding questions on basic programming techniques are provided in native language, to which users can provide solutions in their native language; hence allowing students to focus on good logic and problem-solving skills as their launchpad into the world of writing code. Their solution in the native language will then be the input to a model which will convert this statement into python code in their natural language (educational) and English (to execute). This in turn helps the users visualise the translation of logic to code.

This model uses Parts of Speech (PoS) tagging for the native language [2] as the base, along with language semantics words like variables, quantities, conditional words, and actions. A stemmer [15] for the same language is used to find the relational operation to be done in the conditional statement (example, greater than, not greater than).

A large amount of procedural data was obtained from wiki How [26] and was translated to Kannada. This data was then used to develop and evaluate a model that could identify conditional statements in native language plain text and convert it to pythonic code. The flowchart figure 3 represents the platform to be achieved.

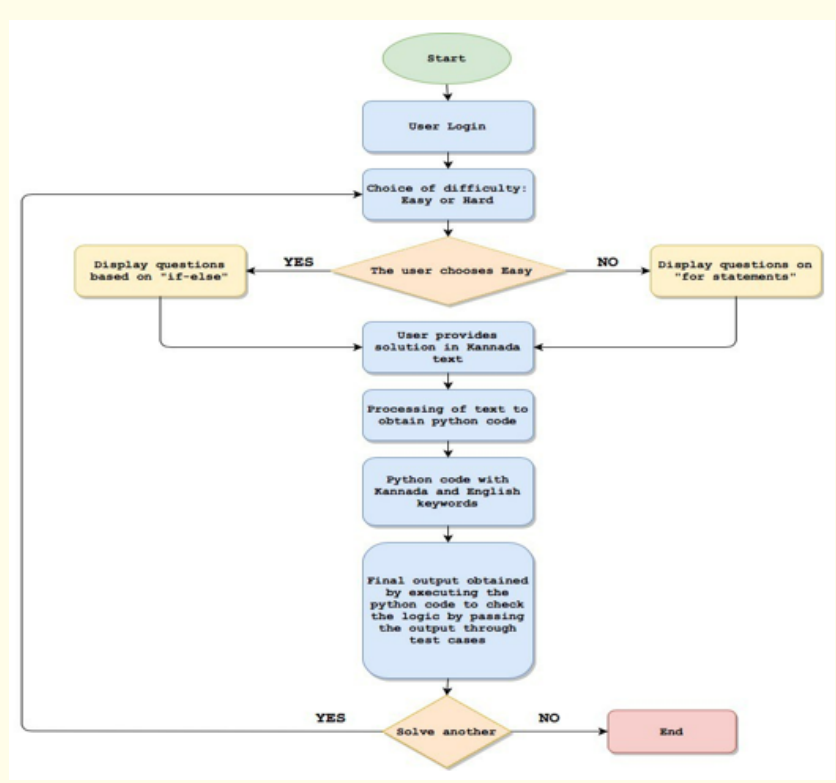

Figure 3: Platform aimed to be achieved. 


\section{Materials and Methods}

This model is based mainly on Parts of Speech tagging. It uses the concept of transfer learning using fast.ai's ULMFit [12]. The RNN model with pre-defined weights requires a dataset for training the model for Parts of Speech tagging. The dataset used for this is called Kasthuri. It contains various words in Kannada vocabulary and the corresponding tag. A snippet from Kasthuri dataset is shown in table 1 [1].

A stemmer $[20,23]$ was also used to extract the root word from conjunctions that usually carry the conditional word. It is a static model that uses dictionaries corresponding to various tenses in Kannada. Each of these dictionaries consist of various suffixes based on which stemming is done.

\begin{tabular}{|c|c|c|}
\hline Kannada & Translation & Output \\
\hline లుడుడి & Udupi & $\mathrm{NN}$ \\
\hline భారాత్ర & Bhaarata & $\mathrm{NN}$ \\
\hline శేనాణట్రెదద & Karnataka & $\mathrm{NN}$ \\
\hline ఒందు & One & QC \\
\hline జిల్లి & District & NN \\
\hline * & $*$ & SYM \\
\hline విఖ్యాతే & Popular & $\mathrm{JJ}$ \\
\hline ముందిర & Temple & $\mathrm{NN}$ \\
\hline कரగ่。 & And & $\mathrm{CC}$ \\
\hline బందిరువుదు & Has come & VM \\
\hline
\end{tabular}

Figure a: Snippet from the Kasturi dataset. were collected from Kannada news websites and 32000 Wikipedia Articles, which have been cleaned. Table 1 represents the sample headlines present in the iNLTK library and table 1 displays the statistics of various topics in the iNLTK library.

\begin{tabular}{|l|c|}
\hline Headlines & Category Split \\
\hline 5114 & Entertainment $52 \%$ \\
Unique values & Sports $36 \%$ \\
& Other $12 \%$ \\
\hline
\end{tabular}

Table 1: Statistics of iNLTK dataset used in this project [9].

To determine the general pattern in conditional statements, the procedural data obtained from wiki How [26] pages were translated to get a dataset of Kannada procedural text, as shown in figure 4.

\begin{tabular}{|c|c|c|c|}
\hline & $\begin{array}{l}\text { News Headline in } \\
\text { Kannada }\end{array}$ & English Translation & Category \\
\hline 1 & 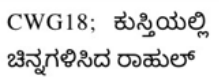 & $\begin{array}{l}\text { "CWG18; Rahul, who won a gold } \\
\text { He's Sushil Kumar" }\end{array}$ & Sports \\
\hline 2 & 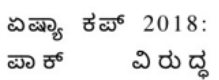 & $\begin{array}{l}\text { "Asia cup 2018: Is Rohit's Army } \\
\text { ready to roar against the Pak....?" }\end{array}$ & Sports \\
\hline 3 & 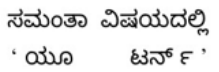 & $\begin{array}{l}\text { "Naaga Chaitanya who took a "U } \\
\text { turn' in the matter of Samanta." }\end{array}$ & Entertainment \\
\hline
\end{tabular}

Figure b: Samples headlines from the dataset.

Apart from this, the iNLTK [9] libraries that were used and referenced in this project, uses 6300 news article headlines, which

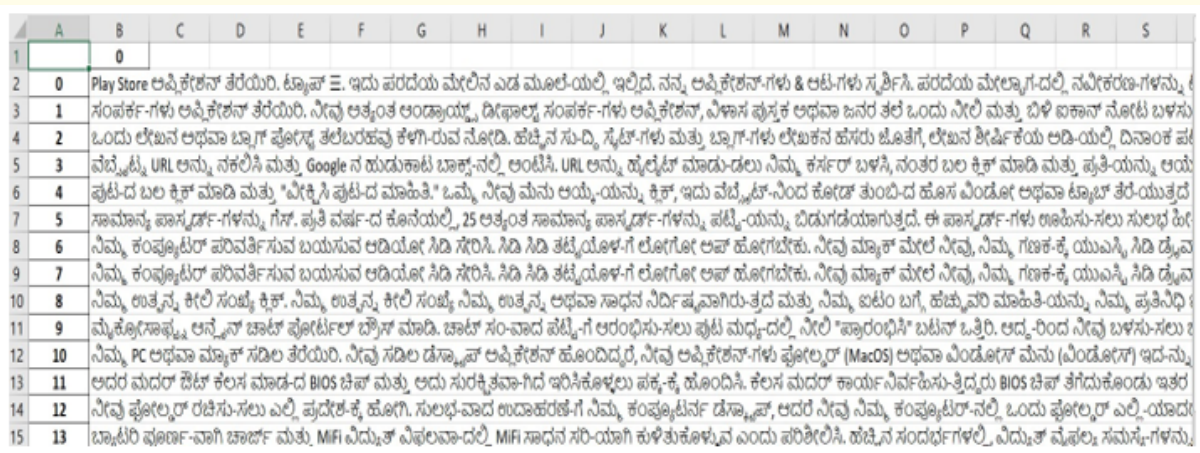

Figure 4: Platform aimed to be achieved. 


\section{Results and Discussion}

Previous work and theoretical model

In the space of providing an interface that accepts Natural Language inputs in Indic languages and translates that into executable Python, our research yielded no such previous work. Most of the applications previously developed cater to the space of text translators like Google Translate [25], OmTransliterator [29], or, work in the space of code to code translation like Facebook's work on Transcoding [28] or in the space of building code parsers that translate Kannada script into an executable script [30]. While all of the previous work helped take steps in the right direction to solve the challenge of making code more accessible, we found that our utility had little specific predecessors in terms of translation Natural languages (like Kannada) into executable code.

The model's main aim is to recognise various programming constructs in plain text and convert them into python code. As of now, the model can recognise conditional statements in Kannada text and convert them into python code.

There are various methods of achieving this. There are existing models to recognise conditional statements in English [19], these models could be used on Kannada text by translating the entire text to English first. Google Translate [25] was one of the options. However, given that we wanted to perform the entire task in the chosen native language (Kannada), performing this translation up front was not a viable design choice for us.

The second method is to use natural language processing techniques [4] by translating each Kannada word to English word and applying an English Parts of Speech tagger model [17] on it. But this would not be an efficient method because of the difference in semantics of the two languages. A single word in Kannada may translate to more than two words (containing stop words) in English.

\section{‘ळె2్జిద్దరం’’}

Figure 5: Kannada word for "Greater than".

For example, the Kannada word shown in figure 5, is a single word which translates to "greater than" in English. Hence the word which should be tagged as 'verb' will have two tags. Since there were not any natural language models for Kannada, the first target was to build a good model for Kannada.

\section{Parts of speech tagger for Kannada}

PoS tagging on Indian languages, especially Dravidian Languages is a difficult task due to the unavailability of annotated data for these languages. Very little work has been done on Kannada because of the scarcity of good quality annotated data. The recent works in PoS tagging on Kannada have been done with traditional ML techniques like HMM, CRF or SVM [5,14]. PoS tagging was necessary in this case to recognise conditions, actions, variables, and quantities.

The model created was inspired by the backend implementation of the iNLTK [9] libraries to handle use cases in Kannada. The tokenizer and the base model used to perform transfer learning was sourced from the iNLTK [9] codebase. On top of this, a classifier was built using the fast.ai framework [13] that facilitates simple APIs to build a language model and a subsequent classifier model. iNLTK [9] stands for natural language toolkit for Indic languages. It is an open-source Deep Learning library built on top of PyTorch [27] in python which aims to provide out of the box support for various NLP tasks. As of the date of this work, iNLTK [9] library has natural language processing tools for Kannada along with 11 other languages. It consists of tokenizer which has been trained on Kannada Wikipedia articles and Kannada news headlines to learn the general language domain [9] This tokenizer was used for transfer learning on the fast.ai's ULMFit model, shown in figure 6 [13]. Transfer learning refers to the use of a model that has been trained to solve one problem (such as classifying images containing cats) as the basis to solve some other similar problem (such as classifying images containing dogs) [21]. The neural network used for ULMFit's transfer learning is AWD LSTM. AWD LSTM uses drop connect to prevent overfitting of the LSTM [12]. The ULMFit has the following three steps:

- The LM (Language Model) is trained on a general-domain corpus to capture general features of the language in different layers (here iNLTK's tokenizer).

- $\quad$ The full LM is fine-tuned on target task data (here Kasthuri) using discriminative fine-tuning ('Discr') and slanted triangular learning rates (STLR) to learn task-specific features.

- The classifier is fine-tuned on the target task using gradual unfreezing, 'Discr', and STLR to preserve low-level representations and adapt high-level ones [12].

So, the pre-trained model here will be the tokenizer whose last layers will be used for text classification. Figure 7 represents the iNLKTK dataset used for pre-training. The classification process here will be PoS tagging, trained using the Kasthuri dataset, shown in figure $c$ [1]. 


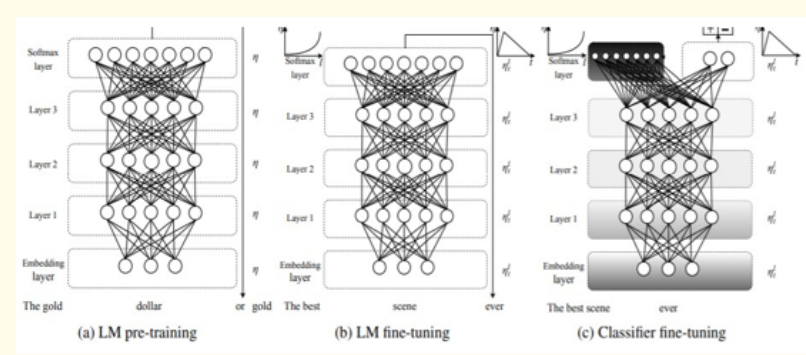

Figure 6: ULMFit on which the POS tagger for Kannada was built [12].

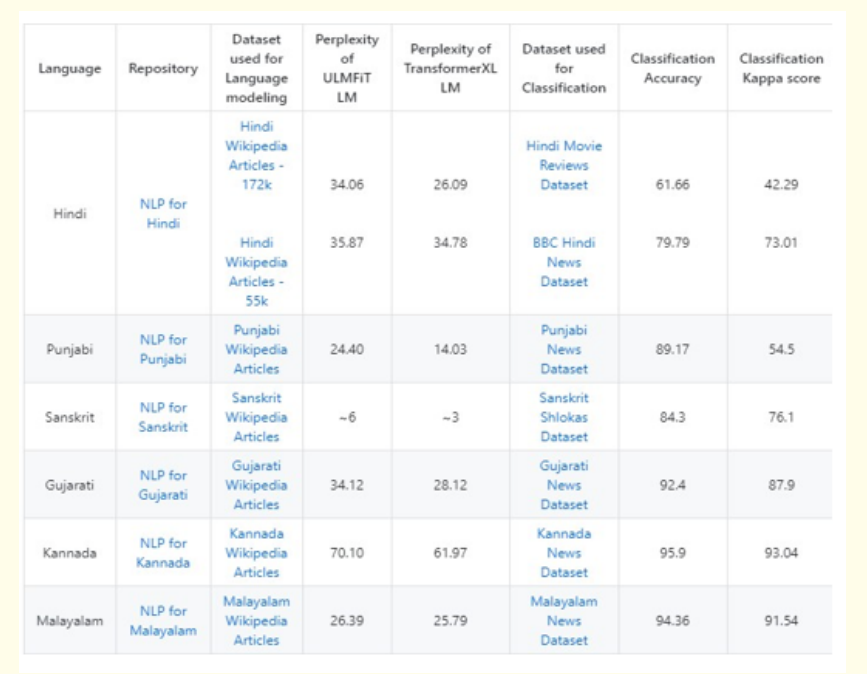

Figure 7: iNLTK' Kannada tokenizer used for LM pre-training of the ULMFit [9].

\begin{tabular}{|c|c|c|}
\hline Kannada & Translation & Output \\
\hline లుడుడి & Udupi & $\mathrm{NN}$ \\
\hline భారాతి & Bhaarata & $\mathrm{NN}$ \\
\hline శనాణటీకేదు & Karnataka & $\mathrm{NN}$ \\
\hline ఒందు & One & QC \\
\hline జిల్లి & District & $\mathrm{NN}$ \\
\hline * & * & SYM \\
\hline విఖ్యాతే & Popular & $\mathrm{JJ}$ \\
\hline ముందిరా & Temple & $\mathrm{NN}$ \\
\hline कగగి & And & $\mathrm{CC}$ \\
\hline బందిరుడుదు & Has come & VM \\
\hline
\end{tabular}

Figure c: Kasthuri dataset used for classifier fine tuning of the ULMFit [1].
Thus, using iNLTK's [9] pre-trained model for Kannada tokenizer and fast.ai's [13] transfer learning method (ULMFit for PoS tagging using the Kasthuri dataset), a Kannada PoS tagger was built.

\section{Stemmer for Kannada}

A stemmer was necessary here to recognise relational operations in plain text and determine whether the operation or its opposite had to be performed. The presence of these words indicates that a sentence is a conditional sentence.

For example, words in figure 8, are two Kannada words that stand for 'greater than' and 'not greater than'. Since the root word in Kannada is necessary to determine the operation as 'greater than' and the suffix to determine if it is 'greater than' or 'not greater than', there was the need for a tool to separate root word and the suffix.

\section{'ळ2జ్బిద్దెరి' 'ळజ్బిల్లదిద్దరా'}

Figure 8: Kannada words for 'greater than' and 'not greater than.

The stemmer used here was a static model. It splits the word as root word and suffix by comparison of the given words with a set of suffixes. There are 74 arrays in total used in the model. These arrays are based on their tense as well as Parts of Speech. Each of these arrays consists of the suffixes which indicate a conditional statement [20].

\section{Text analysis (Algorithm)}

The novelty of our algorithm lies in the observation and translation of language patterns in order to template-ize the occurrence of specific parts of speech and using those extracted parts of speech elements to make decisions on what logical elements they represent. All of the previous work referenced in previous sections target different use cases and therefore present no precedence to compare against.

The Kannada procedural text was passed through the stemmer and PoS tagger. The results obtained were analyzed for a common pattern [3].

The common pattern observed was:

- The first verb in the sentence usually indicated a condition.

- The second verb indicates the end of the action.

- The presence of more than two verbs indicate that it is not a simple conditional statement, but it is either and else, else if or nested condition. 
- The first noun in the sentence is the variable.

- The quantities are the terms on which relational operations are performed.

- $\quad$ The word split by the stemmer is the relational operation.

If the root word is same but suffixes are opposite, then it is an else condition. If the root words are different, then it is an else if or nested condition. The presence of words such as the ones in figure 9 and other similar words indicate that it has multiple comparisons in a single conditional statement. The flow of text analysis is shown in figure 10.

: 'అథావా '(Or) and 'మేత్తు'(And)

Figure 9: Kannada words for "Or" and "And".

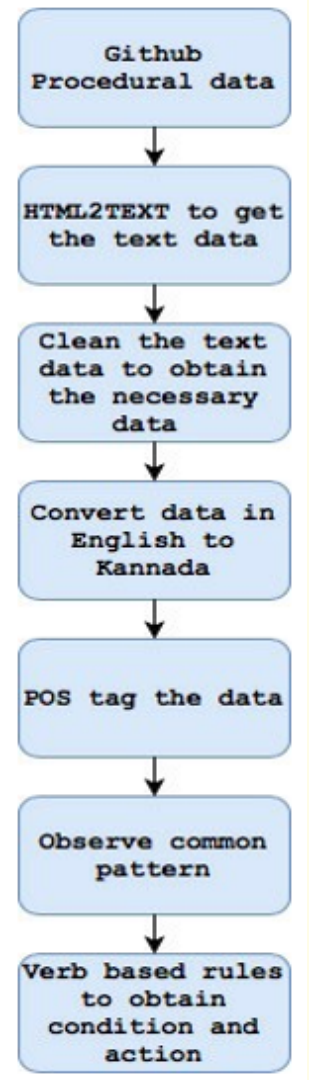

Figure 10: Process used to obtain textual dataset in Kannada for analysis and obtaining verb-based rules.

\section{Displaying in Kannada python code}

The result obtained from above step is converted to Kannada python code by printing the keywords in Kannada and placing the various extracted features such as variables, quantities, conditions and actions the way they would occur in a python programming construct. For example, code in Kannada would look like figure 11.

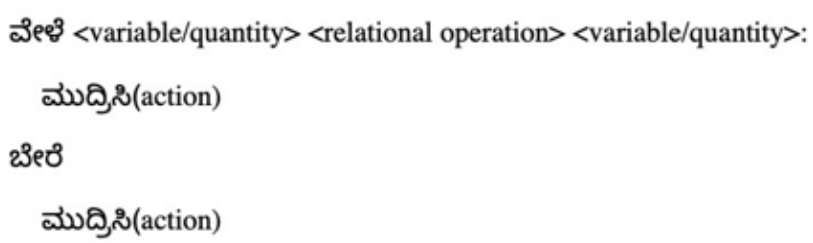

Figure 11: Code Snippet in Kannada.

Displaying code in python

The code in Kannada python-format can be converted to English python code by mapping the keywords in Kannada python to English keywords using a simple dictionary. The above code in python will be translated to the following, as shown in figure 12 .

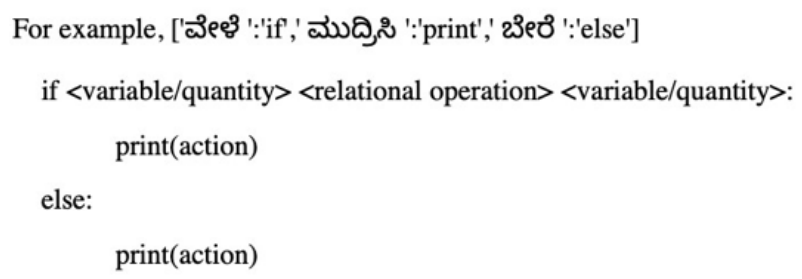

Figure 12: English translation of code with Kannada mapped keywords.

\section{Controlled factors}

The controlling factor in this project is the language semantics. In linguistics, semantics is the study of meaning, as inherent at the levels of words, phrases, sentences, and larger units of discourse. These language semantics remain constant in the project and is useful for tokenizing as well as text analysis.

\section{Methods of regression studied}

To achieve the mentioned goals, various models were used. There was a trial on StanfordNLP [16] for Kannada to achieve 
named entity recognition for Kannada and sentiment analysis for positive and negative tags for conditional words as well.

Named entity recognition for Kannada, using StanfordNLP for Kannada, could not be achieved, due to the lack of training dataset. Hence alternative methods had to be figured out. Kasthuri dataset for PoS tagging in Kannada (which was available on GitHub) was used. With this PoS tagger, good accuracy was obtained to complete this task.

Sentiment analysis [18] for negative and positive tags of words to recognise whether the given relational operation must be performed, or its negation was also tried. This seemed like a good method at the beginning. But later it seemed like this task could be achieved using a simple static stemmer model rather than sentiment analysis which seemed like an over-engineered solution.

Hence after studying various methods, it was possible to build a model to achieve these tasks with a PoS tagger and a static stemmer. The workflow of the entire model is represented in figure 13.

\section{Results}

Overall, based on examination of the model and the training data, the logic entered by the user in a native language (Kannada) gets tagged to python code. Some of the sentences entered by the user were aimed at outright errors and others at inconsistencies. The rules certainly don't exhaust all the errors and inconsistencies found in the training data, but there are enough covering a number of the most common problem that gives some idea as to whether such taxonomic improvements might noticeably lift the tagging accuracy.

The code works fine for if and if-else conditional statements and gives an accuracy of about $87 \%$. So, the words in the sentence are individually sent to the model which does PoS tagging. From there, the verbs nouns and quantity values are extracted. All the verbs are stemmed and the first verb, when stemmed, gives the condition that the user intends to check against. After which, this verb along with the words following it which specify what needs to be done once the condition is satisfied, are picked, and constructed to the structured python code.

After this, few of the Kannada words are mapped to python keywords, is mapped to "if" figure 14, for example and so on.

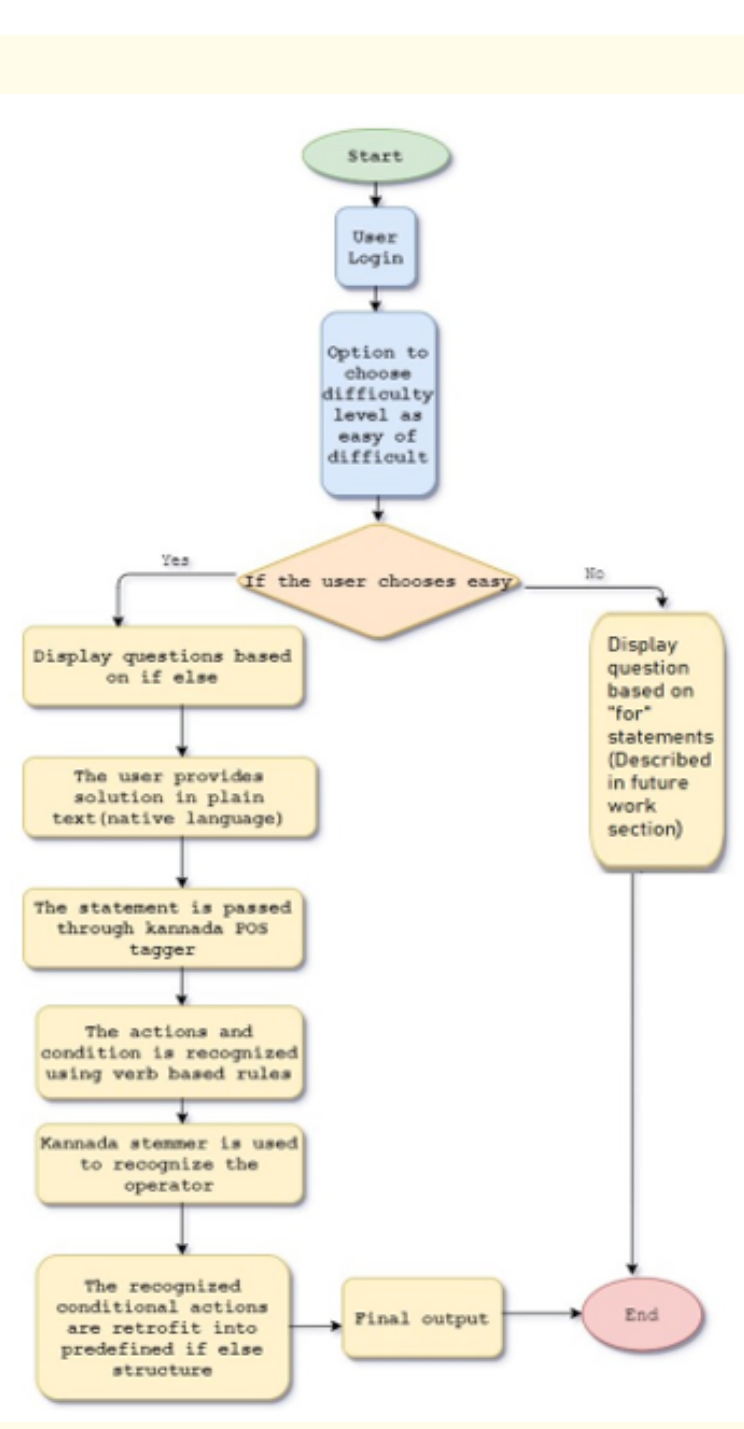

Figure 13: Platform Completed can convert any conditional statement in plain text to python code and execute it to obtain final output.

\section{"విలతి"}

Figure 14: Kannada word for "if".

The code also cleans the text entered by the user to emphasise on the presence of verbs and includes methods to look for opposite 
As of now, recognition and conversion of conditional statements to code have been successful. Further, this model can be extended to convert Kannada statements, with words involving loops and equal-to cases, into their respective codes. This will help the user understand the basic case of iteration and the involved steps of initialisation, condition and updating.

Further, this model can be successfully extended to more Indian languages and hence it would be more accessible to people from all backgrounds. Currently a static synonym check method is used which will be enhanced to become dynamic.

Eventually, the aim is to create a platform to help students learn coding in their choice of native language, wherein the user is expected to provide a suitable logic for the given problem in plain text. The user-interface will have a text editor where the user will provide their logic. The backend which has the NLP Model as an integral part, will then produce python code in both, native language (as a learning exercise) and in English (to execute) which will be compared with the right solution. The user will be notified about their errors and the right code.

With this work, we wish to make coding more accessible and eliminate the language barrier to writing code. Graphical representation of future work on the platform is represented in figure 18.

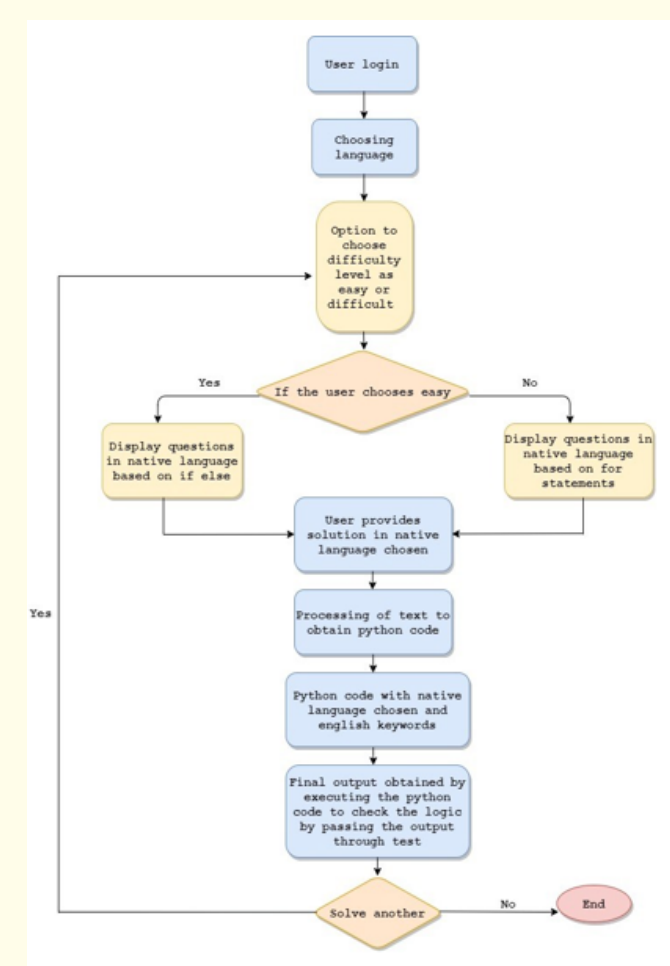

Figure 18: Future work on the Platform to choose any native language to code in.

\section{Acknowledgements}

A short acknowledgement section can be written acknowledging the sources regarding sponsorship and financial support. Acknowledging the contributions of other colleagues who are not included in the authorship of this paper should also be added in this section. If there are no acknowledgements, then this section need not be mentioned in the paper.

\section{Conflict of Interest}

NA.

\section{Bibliography}

1. Adrsh18, Kasthuri dataset.

2. Antony Palesa. "Parts of Speech Tagging for Indian Languages: A Literature Survey". International Journal of Computer Applications 34.8 (2011).

3. Baldwin T., et al. "Automatic Generation of Conditional Diagnostic Guidelines". AMIA Annual Symposium Proceedings 2016 (2017): 295-304.

4. Daniel Jurafsky and James H Martin. "Speech and Language Processing an Introduction to Natural Language Processing, Computational Linguistics, and Speech Recognition" (2019).

5. Deepamala N and P Ramakanth Kumar. "Kannada Stemmer and Its Effect on Kannada Documents Classification" (2015).

6. Erdem Emekligil., et al. "A Bank Information Extraction System Based on Named Entity Recognition with CRFs from Noisy Customer Order Texts in Turkish" (2016).

7. Fang Luo., et al. "Product Named Entity Recognition Using Conditional Random Fields" (2011).

8. Frederic P Miller., et al. "Non-English-Based Programming Languages" (2012).

9. Gaurav Arora, iNLTK, nlp-for-kannada.

10. Hidayat Ur Rahman., et al. "Disease Named Entity Recognition Using Conditional Random Fields".

11. Ivan Ruby., et al. "Natural-Language Neutrality in Programming Languages: Bridging the Knowledge Divide in Software Engineering" (2016).

12. Jeremy Howard and Sebastian Ruder. "Universal Language Model Fine-tuning for Text Classification" (2018).

13. Jeremy Howard., et al. "fastai-A Layered API for Deep Learning" (2020).

14. Ketan Kumar Todi., et al. "Building a Kannada POS Tagger Using Machine Learning and Neural Networks Models” (2018). 
15. M Thangarasu and R Manavalan. "A Literature Review: Stemming Algorithms for Indian Languages" International Journal of Computer Trends and Technology (IJCTT) 4.8 (2013).

16. Manning, Christopher D., et al. "The Stanford CoreNLP Natural Language Processing Toolkit". In Proceedings of 52nd Annual Meeting of the Association for Computational Linguistics: System Demonstrations (2014): 55-60.

17. Raj R., et al. "An enhanced Parts of Speech tagger for English sentence". 9 (2016): 6111-6118.

18. Ramanathan Narayanan., et al. "Sentiment analysis of conditional sentences". In Proceedings of the 2009 Conference on Empirical Methods in Natural Language Processing: Volume 1 - Volume 1 (EMNLP '09). Association for Computational Linguistics, USA (2009): 180-189.

19. Reinhardt Wenzina and Katharina Kaiser. "Identifying Condition-Action Sentences Using a Heuristic-based Information Extraction Method" (2013).

20. Sahana M. shabdhosk.

21. Sebastian Rudder. "Transfer Learning - Machine Learning's Next Frontier" (2017).

22. Stephen Merity., et al. "Regularizing and Optimizing LSTM Language Models” (2017).

23. Suma Bhat. "Statistical Stemming for Kannada" (2013).

24. Vishaal Jatav., et al. "Improving Part-of-Speech Tagging for NLP Pipelines" (2017).

25. Yonghui Wu., et al. "Google's Neural Machine Translation System: Bridging the Gap between Human and Machine Translation" (2016).

26. "WikiHow for procedural data".

27. Zeming., et al. "PyTorch: An Imperative Style, High-Performance Deep Learning Library". Advances in Neural Information Processing Systems 32 (2019): 8024-8035.

28. https://arxiv.org/pdf/2006.03511.pdf

29. "OmTransliterator".

30. https://www.researchgate.net/publication/261013439_ PraNaMa-_Scripting_Language_in_Kannada

\section{Assets from publication with us}

- Prompt Acknowledgement after receiving the article

- Thorough Double blinded peer review

- Rapid Publication

- Issue of Publication Certificate

- High visibility of your Published work

Website: www.actascientific.com/

Submit Article: www.actascientific.com/submission.php

Email us: editor@actascientific.com

Contact us: +919182824667 